\title{
Obtención del relieve digital mediante proyección de luz estructurada en modelos analógicos de extensión
}

\author{
Mariano Cerca ${ }^{1, *}$, Bernardino Barrientos-García², Jorge García-Márquez², \\ Caridad Hernández-Bernal ${ }^{3}$ \\ ${ }^{1}$ Centro de Geociencias, Universidad Nacional Autónoma de México, Campus Juriquilla, \\ CP 76230, Juriquilla, Querétaro, México. \\ ${ }^{2}$ Centro de Investigaciones en Óptica, A. C. \\ Loma del Bosque 115, Apdo. Postal 1-948. C.P. 37000, León, México. \\ ${ }^{3}$ Instituto de Geología, Universidad Nacional Autónoma de México, \\ Ciudad Universitaria, 04510. México, D.F. \\ *mcerca@geociencias.unam.mx
}

\begin{abstract}
Resumen
Se muestran resultados del uso de una técnica de proyección de luz estructurada para obtener un mapa digital del relieve durante la deformación por extensión en modelos analógicos que simulan la parte superior de la corteza terrestre. La técnica para obtener el relieve consiste en la proyección de un patrón de luz estructurada en franjas binarias, luz y sombra, sobre la superficie del modelo. El modelo es deformado progresivamente y se obtiene una fotografía digital de la superficie para cada incremento de deformación. El sistema de deformación es de tipo squeeze-box y consiste de una caja de acrílico dentro de la cual se construyen los modelos usando arena y silicón con diferentes diseños experimentales. Una pared vertical móvil dentro de la caja se desplaza a velocidad constante permitiendo la extensión del modelo. Los resultados obtenidos ilustran la utilidad de las técnicas ópticas para analizar la deformación superficial en los modelos físicos y representar los resultados de manera digital.

Palabras clave: modelos analógicos, extensión, comportamiento mecánico, luz estructurada, proyección de franjas, relieve, análisis de imágenes.

Abstract

We present the results of using a projected structured light technique to obtain a digital topographic map in analogue models of deformation during extension. The analogue models simulate extensional processes occurring in the uppermost part of the earth crust. The technique to obtain the relief consists in the projection of a structured light (binary fringes produced by light and shadows) on the surface of the model. The model is deformed and a digital photograph of the surface is obtained for each deformation increment. The deformation apparatus is squeeze-box type and consists of a Plexiglas box in which models are constructed using materials that simulate the mechanical behavior of the earth crust. A vertical moving wall is displaced within the box at a constant and low velocity allowing the extension of the model. The optical array was constructed in Centro de Investigaciones en Óptica. Results obtained illustrate the convenience of the optical techniques to analyze the surface deformation on the physical experiments.
\end{abstract}

Key words: analogue models, extension, mechanical stratification, structured light, fringe projection, relief, image analysis. 


\section{Introducción}

Mediante la experimentación física de la deformación en modelos a escala construidos con materiales análogos, es posible obtener pistas importantes sobre la dinámica de la deformación en la corteza terrestre. Una de las áreas que se encuentra en desarrollo dentro del modelado analógico es la descripción con precisión de la evolución del relieve durante la deformación. En las últimas dos décadas se han realizado grandes avances en este campo usando diferentes técnicas que incluyen tomografía computarizada de rayos $\mathrm{X}$ (e.g., Colletta et al., 1991; Wilkerson et al., 1992; Schreurs et al., 2001; y referencias incluidas en esos trabajos), escáner láser (e.g., Willingshoffer et al., 2005; Persson et al., 2004) y fotogrametría (Fischer y Keating, 2005). La técnica óptica de proyección de franjas, técnica de campo completo no invasora y de bajo costo, ha sido ampliamente utilizada en la obtención de contornos tridimensionales de diversos objetos estáticos (Indebetouw, 1978; Takeda y Mutoh, 1983; Srinivasan et al., 1984; Windecker y Tiziani, 1995), incluyendo simulación de patrones hidrológicos en medios granulares (Muller et al., 2001). Comparado con un plano de referencia, el monitoreo de la evolución del patrón de luz estructurada permite obtener imágenes consecutivas de diferentes etapas de la deformación de un modelo analógico. Obtener una descripción digital de alta resolución de la evolución de la superficie es de gran importancia para interpretar los resultados y posteriormente trabajar los resultados análogos en un ambiente virtual. En este trabajo se presenta formalmente el desarrollo de la técnica óptica y su aplicación en un modelo sometido a deformación por extensión y con un comportamiento quebradizo.

\section{Arreglo experimental de los modelos}

\subsection{Escalamiento del modelo}

El escalamiento permite que un proceso geológico pueda ser examinado en laboratorio (Ranalli, 2001), en un modelo a escala construido con materiales que presentan un comportamiento mecánico similar al que ocurre en la naturaleza. El escalamiento de los modelos consiste en alcanzar la similitud dinámica, cinemática y geométrica entre los modelos y el fenómeno natural bajo estudio (Hubbert, 1937; Ramberg, 1981; Weijemars y Schmeling, 1986). En este trabajo examinamos la evolución de la superficie de un modelo sometido a extensión, con un comportamiento mecánico quebradizo o con una capa viscosa en la base. Durante la extensión se forman pilares y fosas, bloques elevados y hundidos, respectivamente, que se encuentran limitados por fallas normales (Figura 1). Los procesos que provocan extensión en la corteza han sido extensivamente examinados previamente mediante modelos analógicos en condiciones de gravedad normal y escalada. Por ejemplo, el trabajo de Corti et al. (2003 y referencias incluidas

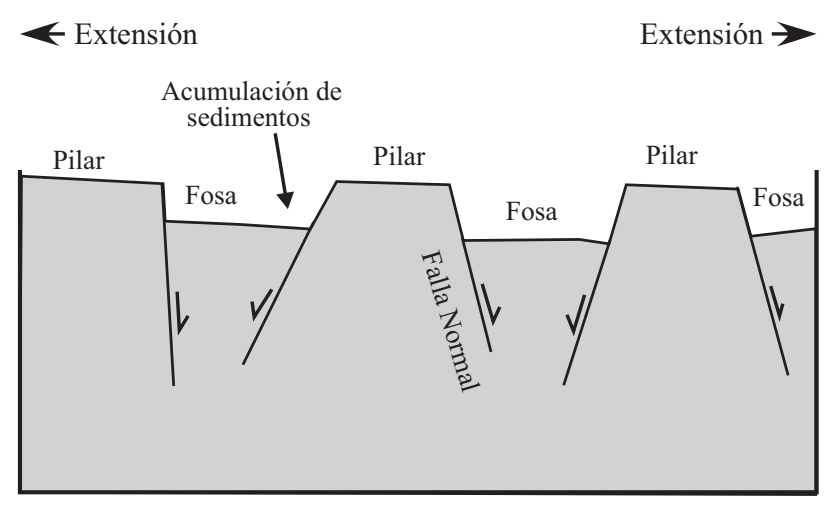

Figura 1. Geometría simplificada en perfil del proceso de extensión continental, en este caso distribuida (Benes y Davy, 1996). No se encuentra a escala.

en ese trabajo) contiene una revisión de los procesos de extensión en modelos analógicos. Hemos llevado a cabo dos variaciones experimentales que simulan extensión en la corteza superior (Figura 2), en condiciones de gravedad normales $g=1$, lo que implica que la ecuación general de escalamiento de esfuerzos (Hubbert, 1937; Ramberg, 1981) se reduce a:

$$
\sigma^{*} \approx 1^{*}
$$

donde $\sigma^{*}$ es la relación de esfuerzos entre el modelo y la naturaleza y $1^{*}$ es la escala geométrica. Los experimentos están diseñados de tal manera que $1 \mathrm{~cm}$ del modelo representa aproximadamente $1 \mathrm{~km}$ en la naturaleza. Los modelos no representan un caso natural específico; el primer caso nos permitió evaluar la capacidad de la técnica de proyección de luz estructurada, mientras que el segundo caso nos permitió comparar los resultados con otros laboratorios similares, mediante un experimento propuesto como guía para la calibración de laboratorios de modelado analógico (Schreurs et al., 2006).

Para modelar la parte superior de la corteza continental de comportamiento mecánico quebradizo (Byerlee, 1978), se utilizó una arena de cuarzo de color blanco, con densidad de $1400 \mathrm{~kg} / \mathrm{m}^{3}$ y tamaño de grano relativamente homogéneo entre 0.3 y $0.4 \mathrm{~mm}$, ángulo de fricción interna de $34^{\circ}$ y cohesión insignificante (Tabla 1). El esfuerzo requerido para activar el desplazamiento de una falla es en gran medida insensible a la composición de la roca (Byerlee, 1978), por lo que la arena se ha considerado un material adecuado para simular la corteza superior quebradiza. La arena tiene un comportamiento mecánico que puede ser expresado mediante el criterio de Mohr-Coulomb:

$$
\tau_{\mathrm{b}}=\sigma \mu(1-\lambda)+c,
$$

donde $\tau_{\mathrm{b}} \mathrm{y} \sigma$ son los esfuerzos de cizalla y normal en el plano de falla, $\mu=\tan \gamma$ es el coeficiente de fricción, $\gamma$ es el ángulo de fricción interna, $\lambda$ es el coeficiente de Hubbert-Rubey 
de presión de fluido, y $c$ es la cohesión. Para simular rocas de la corteza con comportamiento dúctil se utilizan fluidos de alta viscosidad como el silicón SGM36 (Weijemars y Schmeling, 1986) (Tabla 1).

\subsection{Arreglo experimental}

El sistema de deformación utilizado consiste básicamente de una caja de acrílico, en la que se construyen los modelos, y una pared móvil que permite la deformación por acortamiento o extensión de los modelos. Un actuador automatizado con un motor de pasos que permite desplazamientos constantes de entre 1 y $30 \mathrm{~mm} \cdot \mathrm{h}^{-1}$ controla la velocidad y sentido de desplazamiento de la pared móvil durante el experimento.
En el primer experimento, la arena fue añadida mediante tamizado en la caja hasta obtener $5 \mathrm{~cm}$ de espesor y una superficie horizontal. Una hoja de acetato indeformable en la base del modelo se encuentra sujeta a la pared móvil y provoca una discontinuidad de velocidad (VD) que permite localizar las estructuras en la parte central del modelo. En dos corridas experimentales el modelo fue extendido en incrementos de 3 y $6 \%$ hasta $42 \%$, y en una tercera corrida, fue extendido de manera continua hasta $42 \%$ en 50 segundos. En este caso, el resultado de la deformación es independiente de la velocidad de deformación aplicada al modelo.

El segundo experimento explora la influencia durante la deformación extensional de una capa viscosa en la base y es similar al modelo propuesto como calibración entre laboratorios de modelado analógico (Schreurs et al., 2006).

LAMMG-CIO: Arreglo experimental 1

Comportamiento quebradizo

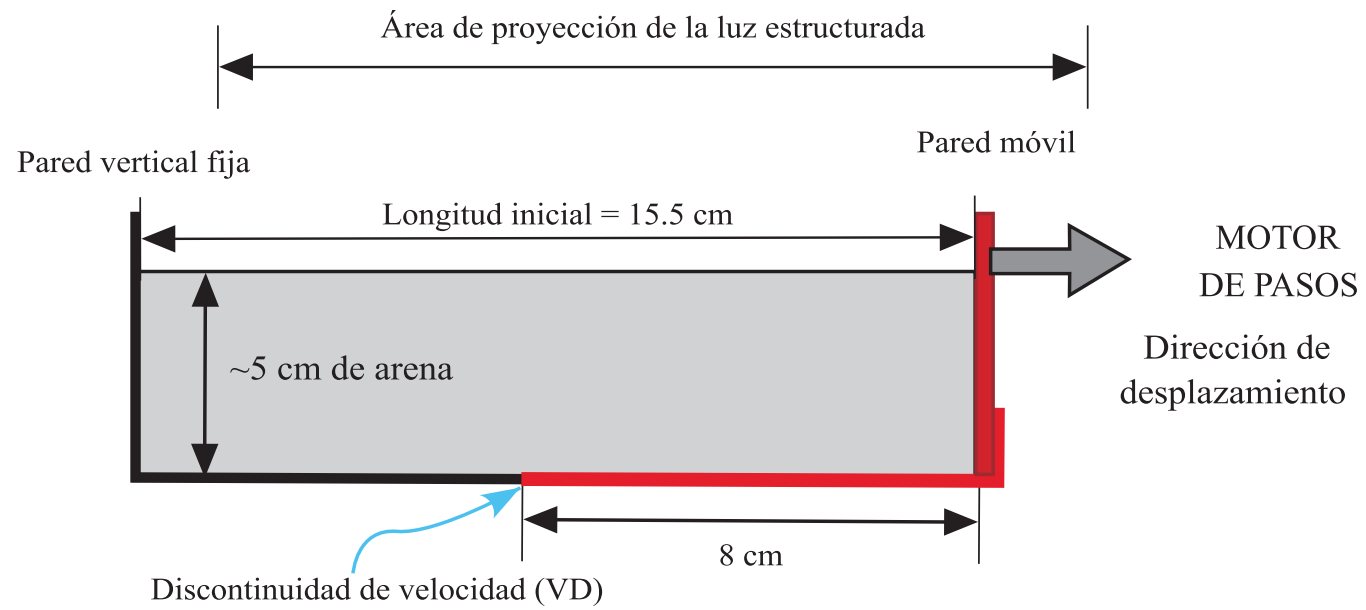

LAMMG-CIO: Arreglo experimental 2

Capa de comportamiento dúctil y débil en la base PDMS SGM 36

ancho del experimento: $15 \mathrm{~cm}$

Pared vertical fija

Pared móvil

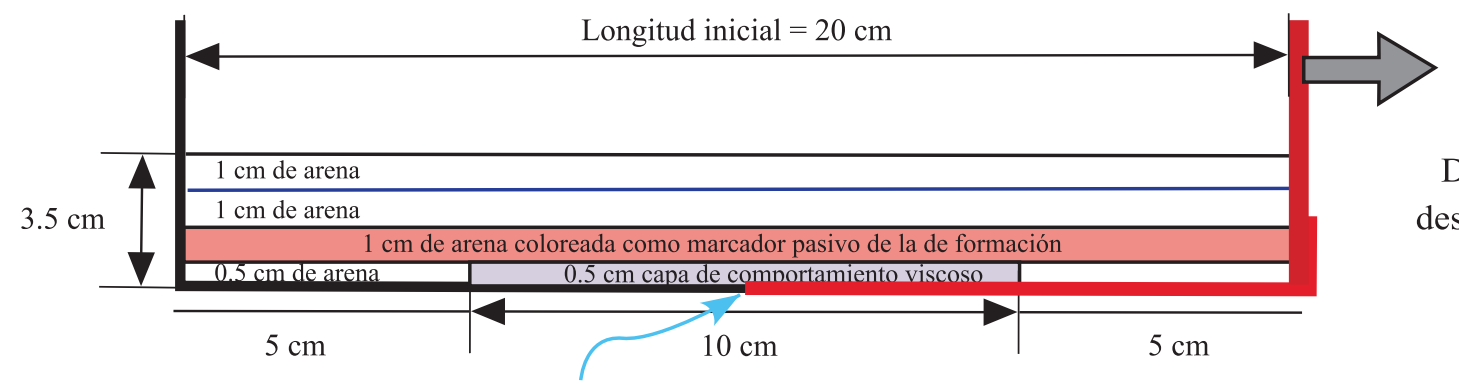

MOTOR

DE PASOS

Dirección de

desplazamiento

Discontinuidad de velocidad (VD)

Figura 2. Arreglo inicial de los experimentos presentados y sistema automatizado de deformación para modelos analógicos. 
Tabla 1. Características de los materiales utilizados como análogos en este trabajo.

\begin{tabular}{lllccc}
\hline Material Análogo & \multicolumn{1}{c}{$\begin{array}{c}\text { Densidad } \\
\left(\mathbf{k g} \cdot \mathbf{m}^{-3}\right)\end{array}$} & \multicolumn{1}{c}{$\begin{array}{c}\text { Comportamiento } \\
\text { mecánico }\end{array}$} & $\begin{array}{c}\text { Angulo de fricción } \\
\text { interna }\end{array}$ & $\begin{array}{c}\text { Cohesión } \\
(\mathbf{P a})\end{array}$ & $\begin{array}{c}\text { Material natural } \\
\text { simulado }\end{array}$ \\
\hline Arena de cuarzo & 1,400 & Quebradizo & $34^{\circ}$ & 80 & Corteza superior \\
Silicón SGM36 & 965 & Viscoso con $\eta=5 \times 10^{4} \mathrm{~Pa} \cdot \mathrm{s}$ & -- & -- & Capa viscosa débil \\
\hline
\end{tabular}

$\eta=$ viscosidad efectiva, valor aproximado en la tasa de deformación utilizada en los experimentos.

Para este experimento se añadió en la base una capa de silicón SGM36 con una viscosidad dinámica de $\eta=5 \times 10^{4}$ Pa s y densidad alrededor de $965 \mathrm{~kg} \cdot \mathrm{m}^{-3}$, con un comportamiento de potencias (power-law) cercano al newtoniano en el intervalo de tasas de deformación utilizado en los experimentos (Weijemars y Schmeling, 1986). El modelo fue extendido con una velocidad constante de $2.5 \mathrm{~cm} \cdot \mathrm{h}^{-1}$ durante 150 minutos. En la Figura 2 se muestra un esquema del arreglo inicial utilizado.

\section{Obtencion del relieve mediante la técnica de proyección de luz estructurada}

El arreglo experimental para analizar la deformación superficial obteniendo un mapa digital de relieve y sus cambios durante la deformación, se llevó a cabo en el Centro de Investigaciones en Óptica, CIO, A. C. El sistema óptico utilizado requiere de un proyector de computadora, un sistema de adquisición de imágenes (cámara digital tipo CCD o CMOS), y un plano de referencia, en este caso la superficie horizontal inicial de la arena (Figura 3). El arreglo óptico está basado en la técnica de proyección de luz estructurada (proyección de franjas) utilizada para medir la componente vertical de desplazamiento de una superficie (Barrientos et al., 2004). Sobre la superficie del modelo se proyectan franjas paralelas binarias de periodo $p$ mediante el proyector, ver Figura 3 (b) y (c). Cuando las franjas son proyectadas sobre una superficie de referencia plana, el período de las franjas sobre la superficie es constante en cualquier punto. Dichas franjas se pueden representar matemáticamente mediante una serie de Fourier,

$$
f(x)=\sum_{n=0}^{\infty} c_{n} \cos \left(\frac{2 \pi}{p} n x\right)
$$

donde $c_{n}$ son los coeficientes de Fourier y $n$ es un número entero..

Sin embargo, es posible referirnos sólo a los dos primeros términos de la serie sin que esto afecte el análisis, ya que las demás componentes armónicas pueden ser filtradas en el espacio de las frecuencias, como se mostrará más delante. Por lo tanto, la rejilla de referencia toma la siguiente forma,

$$
f(x, y)=a(x, y)+b(x, y) \cos \left(\frac{2 \pi}{p} x\right),
$$

donde por comparación con interferometría, $a(x, y)$ representa el nivel de iluminación de fondo y $b(x, y)$ la visibilidad o contraste de las franjas. Cuando este patrón de franjas es registrado mediante una cámara $\mathrm{CCD}$, debido a efectos de perspectiva, el período deja de ser constante y varía respecto a las coordenadas $x, y$. Entonces el nivel de intensidad registrado por el CCD para una imagen modulada con franjas es

$$
I(x, y)=a(x, y)+b(x, y) \cos \left(\frac{2 \pi}{p(x, y)} x\right),
$$

donde se ha omitido un factor de proporcionalidad que toma en cuenta la conversión a unidades de niveles de gris de las imágenes grabadas, es decir la conversión de formato analógico (en voltios) del sensor del CCD al formato digital (de 0-255 niveles de gris, donde el cero corresponde al negro y el 255 al blanco). Esta operación la realiza automáticamente el software de control que viene con la cámara.

La ecuación (5) puede ser equivalentemente representada como

$$
I(x, y)=a(x, y)+b(x, y) \cos \left(\frac{2 \pi}{p} x+\theta(x, y)\right),
$$

donde $\theta(x, y)$ es el cambio en la fase de las franjas debida a efectos de perspectiva. Este término de fase también toma en cuenta las variaciones en el período debido a las aberraciones introducidas por el sistema formador de imágenes del CCD.

Cuando el modelo es sometido a esfuerzos, su superficie se deforma y su topografía varía de un punto a otro, es decir aparece una distribución de alturas sobre la superficie plana. Esto equivale a introducir una nueva variación del período local $p(x, y)$. Este efecto se puede notar en el video anexo (<<http://www.geociencias.unam. $\mathrm{mx} / \sim$ alaniz/ $\mathrm{SGM} /$ suplementos/cercaVideo1.avi $>>$ ) y en las imágenes obtenidas con la cámara CCD. Tomando en cuenta dicho efecto, la ecuación (6) se transforma en:

$I(x, y)=a(x, y)+b(x, y) \cos \left(\frac{2 \pi}{p} x+\theta(x, y)+\phi(x, y)\right)$,

donde $\phi(x, y)$ es la contribución a la fase de las franjas debido a las variaciones en altura de la superficie del modelo durante la deformación. En la ecuación (7) se ha asumido que el término de fase debido a perspectiva, $\theta(x, y)$, permanece sin cambios. Esto sucede así siempre y cuando 
a)

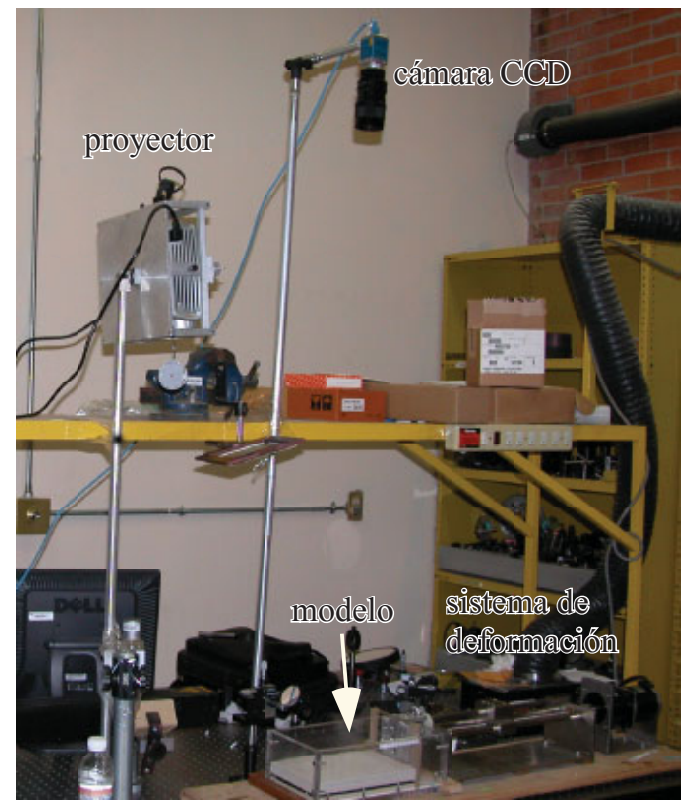

b)
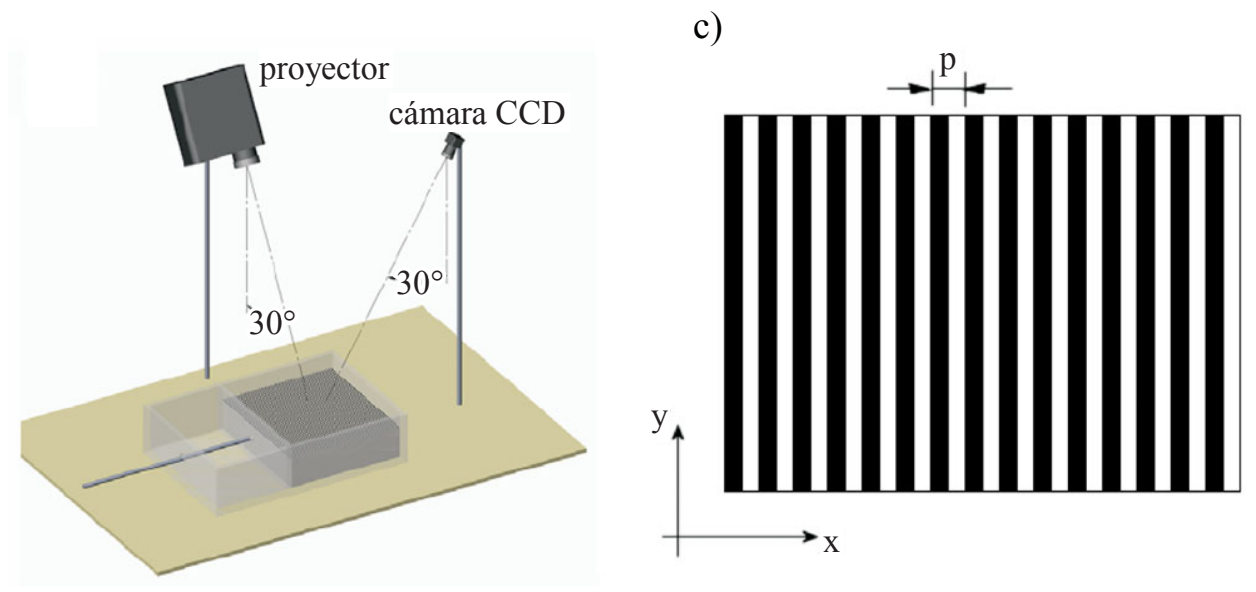

Figura 3. a) Proyección de las franjas binarias sobre la superficie inicial del modelo en la serie experimental 1. b). El arreglo experimental utilizado para el experimento de extensión. c) Franjas proyectadas sobre una superficie plana horizontal. El proyector y la cámara CCD tienen un ángulo de $30^{\circ}$ con respecto a la normal a la superficie del modelo. La pared móvil se retira lentamente a velocidad constante y la arena avanza modulando las franjas proyectadas.

los desplazamientos (o deformaciones) de la superficie sean del orden de unos pocos períodos (Barrientos et al., 2004). Del arreglo geométrico presentado en la Figura 4, podemos encontrar la relación entre $\phi(x, y)$ y la distribución de desplazamientos verticales $h(x, y)$ en la forma siguiente. Suponiendo que la deformación aplicada por la pared móvil sobre la arena provoca un cambio en el relieve de la superficie de uno plano al indicado por $\mathrm{ABCDE}$, entonces los cambios de altura de la superficie modifican la posición de las franjas proyectadas en la dirección $x$. Por ejemplo, la posición de la franja binaria que inicialmente caería en B para una superficie plana, ahora cae en D debido al cambio en altura $\mathrm{BC}$, visto desde la dirección de observación. Como el desplazamiento lateral de la franja es $\mathrm{CD}$, enton- ces en esa posición el período de la rejilla es modificado o equivalentemente la fase de la rejilla binaria en ese punto también lo es. Considerando que un desplazamiento lateral igual a un período de la rejilla proyectada, $p$, equivale a un cambio de fase de $2 \pi$ rad de la rejilla proyectada, entonces el cambio de fase debido a un desplazamiento lateral CD es $\phi(x, y)=\overline{C D}(2 \pi) p^{-1}$. Considerando el triángulo $\mathrm{BCD}$, se observa que la variación de altura $h(x, y)=\overline{B C}$ está dada por:

$$
h(x, y)=\frac{\phi(x, y)}{2 \pi} \frac{p}{\tan \alpha},
$$

donde $\alpha$ es el ángulo medio entre la dirección de observación y la dirección de iluminación. En la ecuación (8) se supone que la distancia de observación es mucho más grande que 


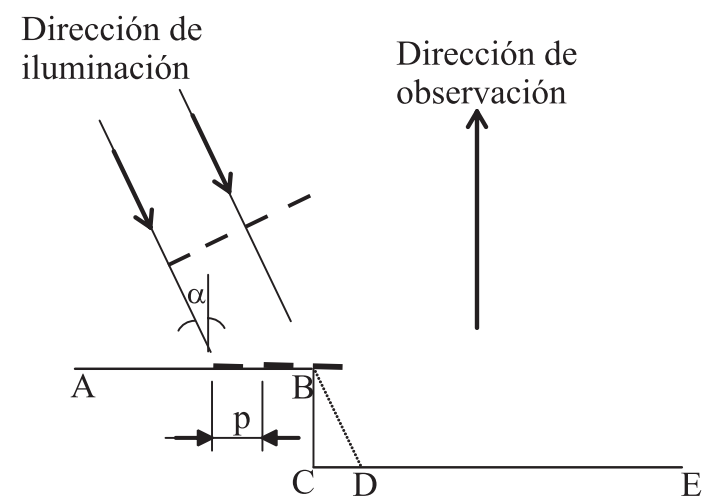

Figura 4. Geometría utilizada en el cálculo del desplazamiento vertical $h(x, y)$. Cuando el objeto se deforma, el punto B se desplaza hasta C. Esto origina que la posición horizontal de la franja que caería en $\mathrm{B}$ se desplace horizontalmente hasta $\mathrm{D}$, visto desde la dirección de observación. El eje $\mathrm{y}$ apunta hacia fuera de la hoja.

las dimensiones de la región observada, condición que se cumple en el arreglo experimental. Además, como los parámetros $p$ y $\alpha$ pueden ser medidos directamente del arreglo experimental, entonces el desplazamiento vertical $h(x, y)$ puede ser obtenido una vez que se conoce el término de fase $\phi(x, y)$. Este término puede ser conocido mediante la resta de los argumentos de las Ecs. (6) y (7):

$$
\phi(x, y)=\left(\frac{2 \pi}{p} x+\theta+\phi\right)-\left(\frac{2 \pi}{p} x+\theta\right) .
$$

Como se puede ver, después de tomar la diferencia de los términos de fase de la imagen de referencia y una imagen con deformación, las contribuciones de los términos por perspectiva, $\theta(x, y)$ y portadora, $(2 \pi) \mathrm{p}^{-1}$, resultan totalmente compensados en el resultado final.

Los argumentos de las funciones coseno en las Ecs. (6) y (7) pueden ser calculados mediante el método de Fourier (Takeda y Mutoh, 1983) a partir de imágenes con franjas del objeto bajo prueba. Para ello, la ecuación (7), por ejemplo, se rescribe de la siguiente forma:

$$
\begin{aligned}
& I(x, y)=a(x, y)+\frac{1}{2} b(x, y) \exp \left(\frac{i 2 \pi}{p} x\right) \exp (i g(x, y))+ \\
& \frac{1}{2} b^{*}(x, y) \exp \left(\frac{-i 2 \pi}{p} x\right) \exp (-i g(x, y))
\end{aligned}
$$

donde $g(x, y)=\theta(x, y)+\phi(x, y)$, el asterisco denota la operación de complejo conjugado e $i=\sqrt{-1}$. Para calcular el término $g(x, y)$, que es el término que contiene el término de interés $\phi(x, y)$, tomamos la transformada de Fourier de la ecuación (10). Para esto, recordamos la propiedad de traslación en el espacio de Fourier,

$$
\mathfrak{I}\left\{f(x) \exp \left(i 2 \pi u_{0} x\right)\right\}=F\left(u-u_{0}\right),
$$

donde $\mathfrak{I}$ representa al operador transformada de Fourier, $u_{0}$ es una frecuencia portadora y $F(u)=\mathfrak{I}\{f(x)\}$, con $u$ como
Cerca et al.

frecuencia espacial. Por lo tanto, la transformada de Fourier de la ecuación (10) produce,

$$
I_{F}(u, v)=A(u, v)+B\left(u-u_{0}\right)+B^{*}\left(u+u_{0}\right),
$$

donde $u, v$ son las coordenadas en el espacio de Fourier, $u_{0}=(2 \pi) p^{-1}, A(u, v)=\mathfrak{I}\{\mathrm{a}(\mathrm{x}, \mathrm{y})\}$ y $B(u, v)=\mathfrak{I}\{1 / 2 b(x, y) \exp$ $(i g(x, y))\}$. El primer término del lado derecho de la ecuación (12) es un término de iluminación de fondo cuyas frecuencias son bajas, es decir su variación espacial es casi nula, y por lo tanto aparece en el espacio de Fourier centrado en $u=0$ y con un ancho más pequeño que $u_{0}$, como se observa en la Figura 5. Los otros dos términos aparecen centrados en $u=u_{0}$, y su magnitud es simétrica. Los términos de mayor frecuencia en la serie de Fourier de la ecuación (4), aparecen centrados en $2 u_{0}, 3 u_{0}$, etc. Sin embargo, su magnitud es relativamente baja y pueden ser removidos como se muestra a continuación. Al aplicar un filtro pasabandas (señalado en línea punteada en la Figura 5), de tal forma que sólo se deje pasar al lóbulo centrado en $u_{0}$, la señal representada por la ecuación (12) se modifica a

$$
I_{F}(u, v)=B\left(u-u_{0}\right) .
$$

Si se toma la transformada inversa de Fourier del resultado dado por la ecuación (13), se obtiene lo siguiente,

$$
\begin{aligned}
\mathfrak{J}^{-1}\left\{B\left(u-u_{0}, v\right)\right\} & =\mathfrak{I}^{-1}\left\{\mathfrak{I}\left\{\frac{1}{2} b(x, y) \exp (i g(x, y))\right\}_{u-u_{0}}\right\} \\
= & \frac{1}{2} b(x, y) \exp (\operatorname{ig}(x, y)) \exp \left(i 2 \pi u_{0} x\right) \\
= & R(x, y)+i M(x, y),
\end{aligned}
$$

donde

$$
R(x, y)=\frac{1}{2} b(x, y) \cos \left(2 \pi u_{0} x+g(x, y)\right)
$$

$\mathrm{y}$

$$
M(x, y)=\frac{1}{2} b(x, y) \sin \left(2 \pi u_{0} x+g(x, y)\right)
$$

Para obtener el segundo paso de la ecuación (14) se hizo uso nuevamente de la propiedad de traslación, ecuación (11).

De las ecuaciones (15) y (16) se puede obtener el término de fase buscado,

$$
\begin{aligned}
2 \pi u_{0} x+g(x, y) & =\frac{2 \pi}{p} x+\theta(x, y)+\phi(x, y) \\
& =\tan ^{-1}\left(\frac{M(x, y)}{R(x, y)}\right)
\end{aligned}
$$

Similarmente, al aplicar este procedimiento a la imagen de referencia, representada por la ecuación (6), y usando la ecuación (9), finalmente se calcula la fase debida solamente a deformación y por tanto se obtiene la distribución de 
Unidades arbitrarias

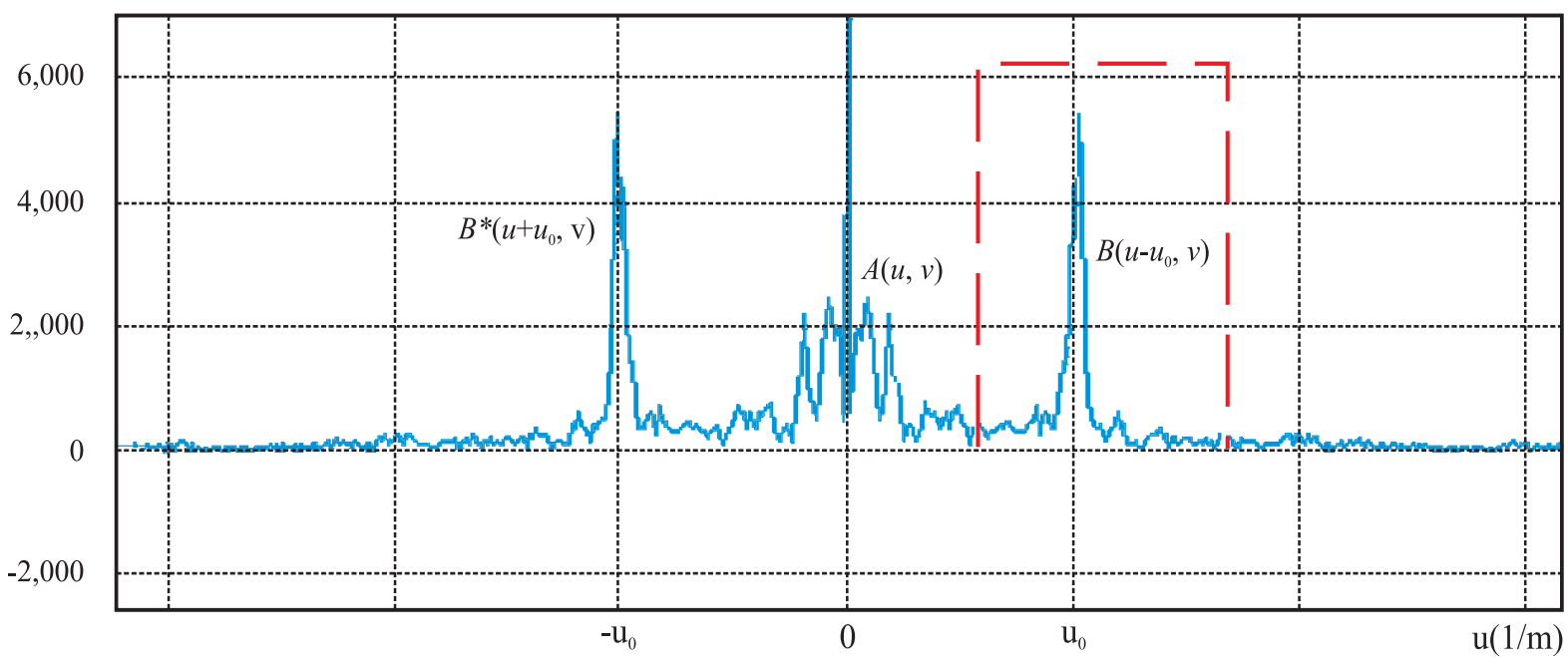

Figura 5. Perfil horizontal de la transformada de Fourier de una imagen con franjas. Las unidades de $u, v$ están dadas en $1 / \mathrm{m}$, cuando las de $x$ y $y$ están en $\mathrm{m}$.

desplazamientos verticales en toda la superficie del modelo mediante la ecuación (8).

Si la deformación es mayor que el periodo $p$, entonces es necesario realizar un proceso de desenvolvimiento de fase al resultado dado por la ecuación (9) (Bryanston-Cross et al, 1994).

A partir de la ecuación (8), se puede apreciar que la resolución del método depende de la selección del periodo y del ángulo de observación. La resolución obtenida en los resultados de este trabajo resulta adecuada para nuestros objetivos pues se encuentra en el intervalo de decenas de micrómetros, mientras que los desplazamientos verticales en los modelos analógicos generalmente fueron del orden de milímetros.

En resumen, para calcular el desplazamiento vertical en modelos analógicos sometidos a deformación, el método requiere los siguientes pasos:

1. Proyectar una imagen con franjas binarias (negras y blancas) que cubra la región de interés. Las franjas proyectadas deben ser paralelas a la pared móvil. Se sugiere un período de 2-3 $\mathrm{mm}$ (medido sobre la superficie) para cambios de elevación totales del orden de 4-6 cm.

2. Grabar una imagen de referencia (Figura 6) cuando la superficie de la arena está inicialmente horizontal o al final añadiendo arena hasta que la superficie sea horizontal.

3. Grabar tantas imágenes como se requieran aplicando un desplazamiento a la pared móvil.

4. Tomar la transformada de Fourier tanto de la imagen de referencia como de las imágenes con deformación. Estas darán origen a nuevas imágenes de datos.

5. A las imágenes de datos resultantes del paso 4 , aplicar un filtro pasabanda adecuado. La forma del filtro bidimensional (a diferencia del unidimensional mostrado en la Figura 5, que fue calculado para una sola línea de datos) puede obtenerse al graficar la magnitud de la transformada de Fourier para cualquier imagen. Este filtro debe cubrir enteramente al lóbulo más cercano al centro. Generalmente, el filtro dimensional es representado mediante un círculo. Este mismo filtro puede ser usado en cualquier otra imagen, logrando con esto la automatización del proceso.

6. A las imágenes resultantes del filtrado del paso 5, tomarles la transformada inversa de Fourier. Esto dará como resultado que cada imagen de datos origine a dos nuevas imágenes: una que contiene la parte real de la transformada inversa de Fourier y otra a su parte imaginaria, las cuales corresponden a $R(x, y)$ y $M(x, y)$ en las ecuaciones $(15)$ y (16), respectivamente.

7. Calcular la fase para cada píxel de cada imagen de acuerdo a la ecuación (17). De esta forma cada imagen original produce una imagen con datos de fase. A esta última imagen de datos se le conoce como mapa de fase.

8. Para conocer la deformación en cualquier instante del experimento, tomar la resta del mapa de fase correspondiente al instante y el mapa de fase de referencia, píxel por píxel.

9. El desplazamiento vertical para cada píxel para una imagen dada puede entonces ser calculado mediante la ecuación (8). La imagen resultante es del tipo de imágenes de aquellas mostradas en la columna derecha de la Figura 7.

\section{Evolución estructural y desarrollo de relieve en los modelos}

En general, la evolución de la deformación en los experimentos presentó un desarrollo similar de fallas con desplazamiento normal que delimitan las fosas y un desarrollo progresivo que causa una zona extendida cada vez más amplia (Figura 7 y 8). El desplazamiento de la discontinuidad de velocidad provoca la migración de la 


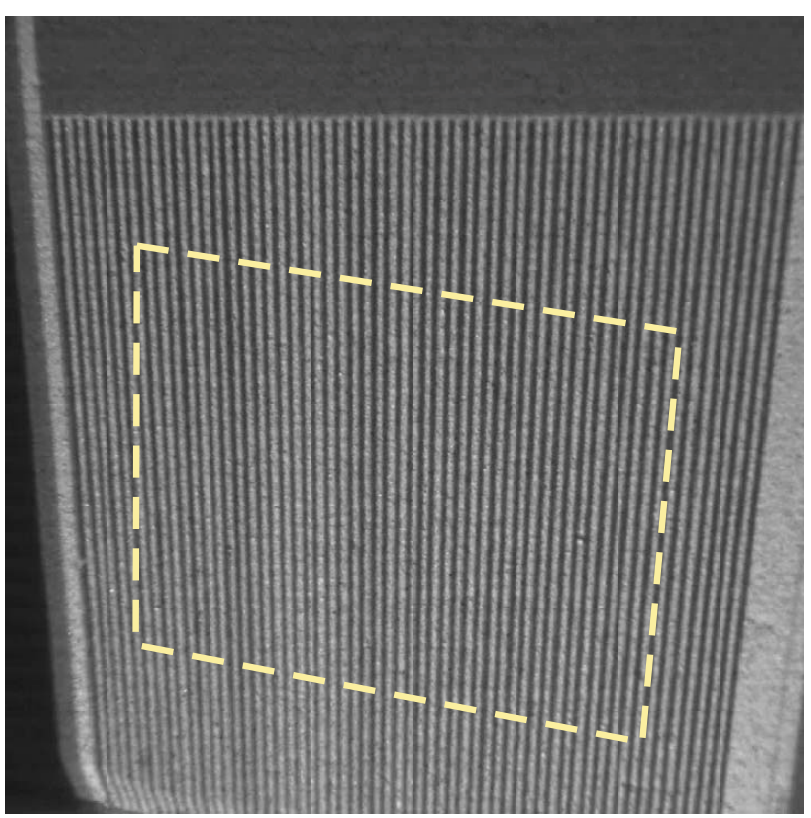

Figura 6. Obtención de un plano de referencia para el cálculo de las alturas.

localización de la deformación y produce fosas con distintos grados de asimetría. En las fotografías presentadas en las Figuras 7 y 8 , las franjas que se observan sobre la superficie de cada modelo corresponden al patrón de luz estructurada proyectado para obtener el mapa digital topográfico en la primera serie experimental. Para documentar la evolución de las estructuras en planta se utilizaron las fotografías obtenidas con la cámara $\mathrm{CCD}$, con un ángulo de $30^{\circ}$ con respecto a la dirección de observación, a intervalos regulares de tiempo (cada 3 minutos). Las imágenes obtenidas son analizadas e interpretadas, en la forma tradicional y con ayuda del mapa topográfico digital, para producir una cartografía de las estructuras formadas en cada intervalo de deformación (Figura 7 a y b). Junto a cada interpretación se presenta también el resultado del mapa digital topográfico (Figura 7 a y b), el cual permite obtener una idea más clara del desarrollo topográfico en la superficie. Además, como material anexo al manuscrito se presentan dos videos que ilustran el desarrollo de la deformación en el modelo disponibles en la página $<<\mathrm{http} / / / \mathrm{www}$.geociencias.unam. $\mathrm{mx} / \sim$ alaniz/SGM/cercaVideos.htm $>>$

En la Figura 7a se muestra la evolución superficial en el primer experimento entre 7 y $21 \%$ de extensión. Antes de $1 \mathrm{~cm}$ de desplazamiento (alrededor de $7 \%$ de extensión) ya se había formado la primera falla normal en el modelo con el bloque de piso, en el lado opuesto a la pared móvil. La posición de esta falla coincide con la posición inicial de la VD (velocity discontinuity) y divide en dos bloques bien definidos el modelo. Hacia los $2 \mathrm{~cm}$ de desplazamiento (14 $\%$ de extensión) se forma la falla con inclinación opuesta que delimita la forma inicial de la fosa. Posteriormente ( $21 \%$ de extensión), la deformación avanza hacia el lado opuesto de la pared en movimiento y produce dos nuevas fallas. Durante este proceso, se observa una zona que se profundiza en el relieve digital en el centro de la fosa y comienza a migrar junto con la VD en la dirección de la pared en movimiento.

En la Figura 7b se muestra la evolución superficial de la deformación entre 28 y $42 \%$ de extensión. En general, se puede observar un ensanchamiento de la zona profunda y una migración menor que en el intervalo anterior entre las dos primeras fallas, además de un aumento en la diferencia de altura entre la parte más alta y baja que va de 3 hasta $4 \mathrm{~cm}$ al final del experimento. El espaciamiento entre las fallas hacia la derecha de la fosa principal también se incrementa progresivamente. Todas las estructuras continuaban activas aún hacia el final del experimento cuando se llegó a un porcentaje de extensión de $42 \%$, la profundidad de la cuenca alcanzó $2.3 \mathrm{~cm}$ y el ancho de la zona extendida aproximadamente $12.1 \mathrm{~cm}$.

Es necesario mencionar que la estructura asimétrica formada durante este experimento pudo haber sido influenciada por efectos de borde causado por la ubicación de la VD cercana a la pared móvil. Una nota de atención en este tipo de experimentos es necesaria para considerar inicialmente la superficie que debe quedar cubierta por la luz estructurada al final del experimento. Al final del experimento, la caja de acrílico fue rellenada con arena hasta llegar a una posición horizontal arbitraria, para obtener un plano de referencia (Figura 6) que permitiera el cálculo de las elevaciones en el modelo.

\subsection{Experimento de calibración (benchmark) entre laboratorios}

En el segundo experimento se reprodujo el experimento propuesto como calibración (benchmark) entre laboratorios de modelado analógico (Schreurs et al., 2006). Un mapa de relieve digital no pudo ser procesado para este experimento debido a que el periodo de franjas y ángulo de observación elegido no fueron óptimos para la técnica de franjas. Sin embargo, los resultados de este experimento son de importancia para la comparación entre laboratorios. Durante el experimento, las primeras fallas se formaron a la izquierda de la VD formando una cuenca angosta. Hacia $7 \%$ de extensión hay más estructuras que en el caso anterior y la extensión se distribuye en una zona que cubre toda el área donde se ubica la capa viscosa (Figura 8). A partir de este momento, la deformación avanza hacia ambos lados de la VD, pero se observa una mejor definición de las estructuras hacia el lado de la pared móvil. En este caso, la deformación vertical es menor y se forma un pilar en la zona central del modelo. La evolución de la deformación en superficie del experimento es similar a los resultados obtenidos por otros laboratorios (ver Schreurs et al., 2006), confirmando que el procedimiento experimental es también similar. Al final del experimento, la fosa a la izquierda del pilar alcanzo 1 

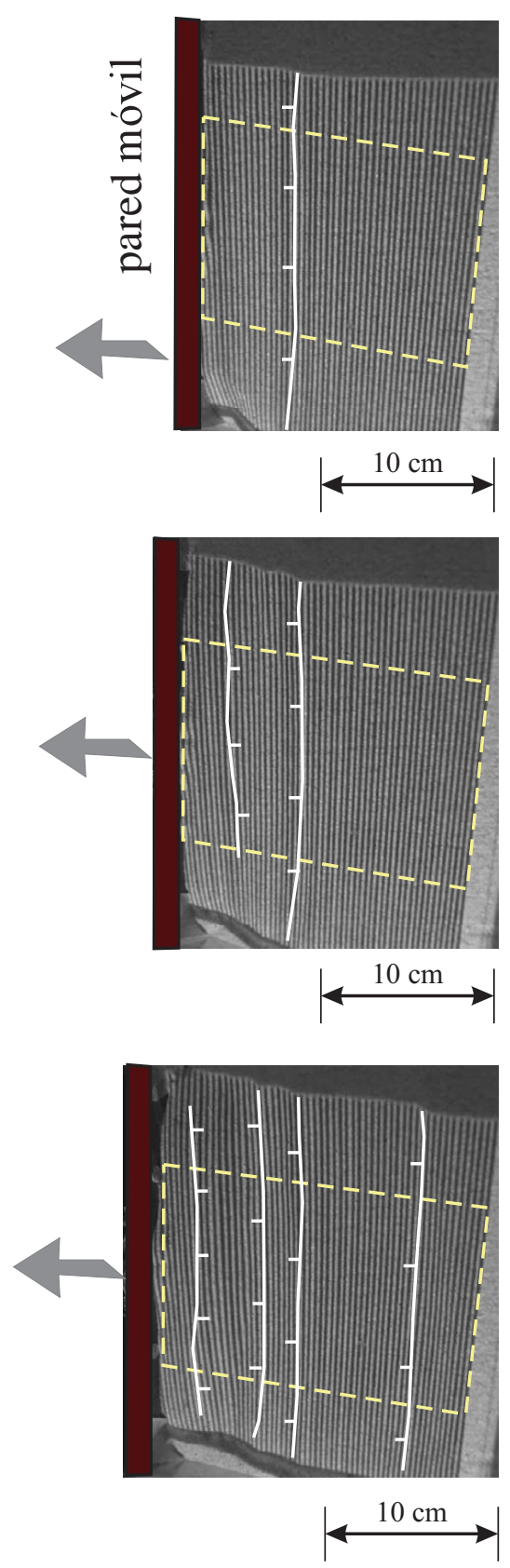

Simbolos

$\perp+$ Falla normal
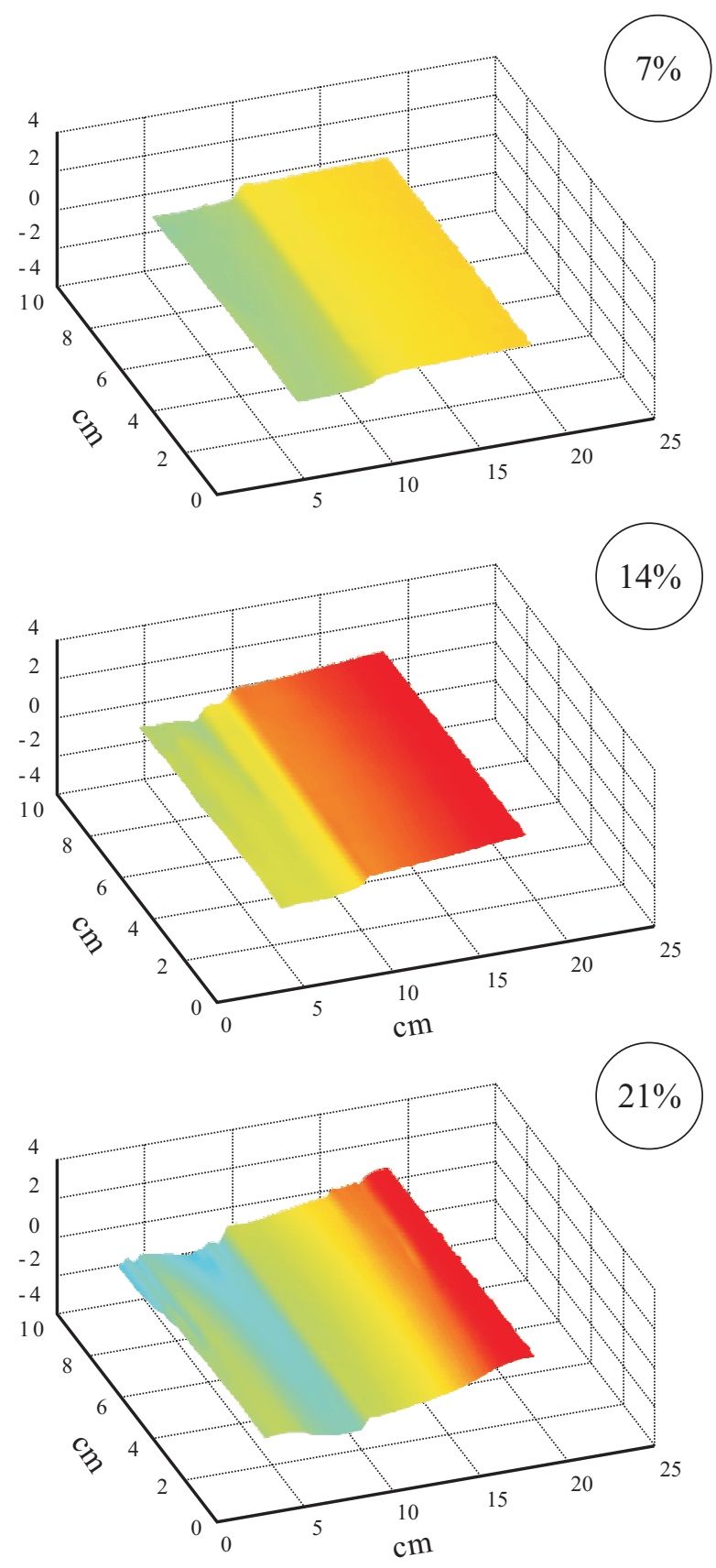

$1 \%$

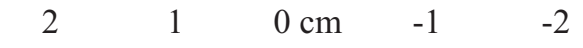

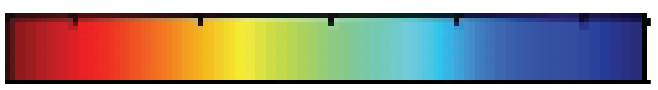

Figura 7a. Evolución de la deformación en el primer experimento, se muestra la interpretación tradicional en las fotografías obtenidas con un ángulo de $30^{\circ}$ y la vista del relieve obtenido en $3 \mathrm{D}$, entre 7 y $21 \%$ de extensión. 

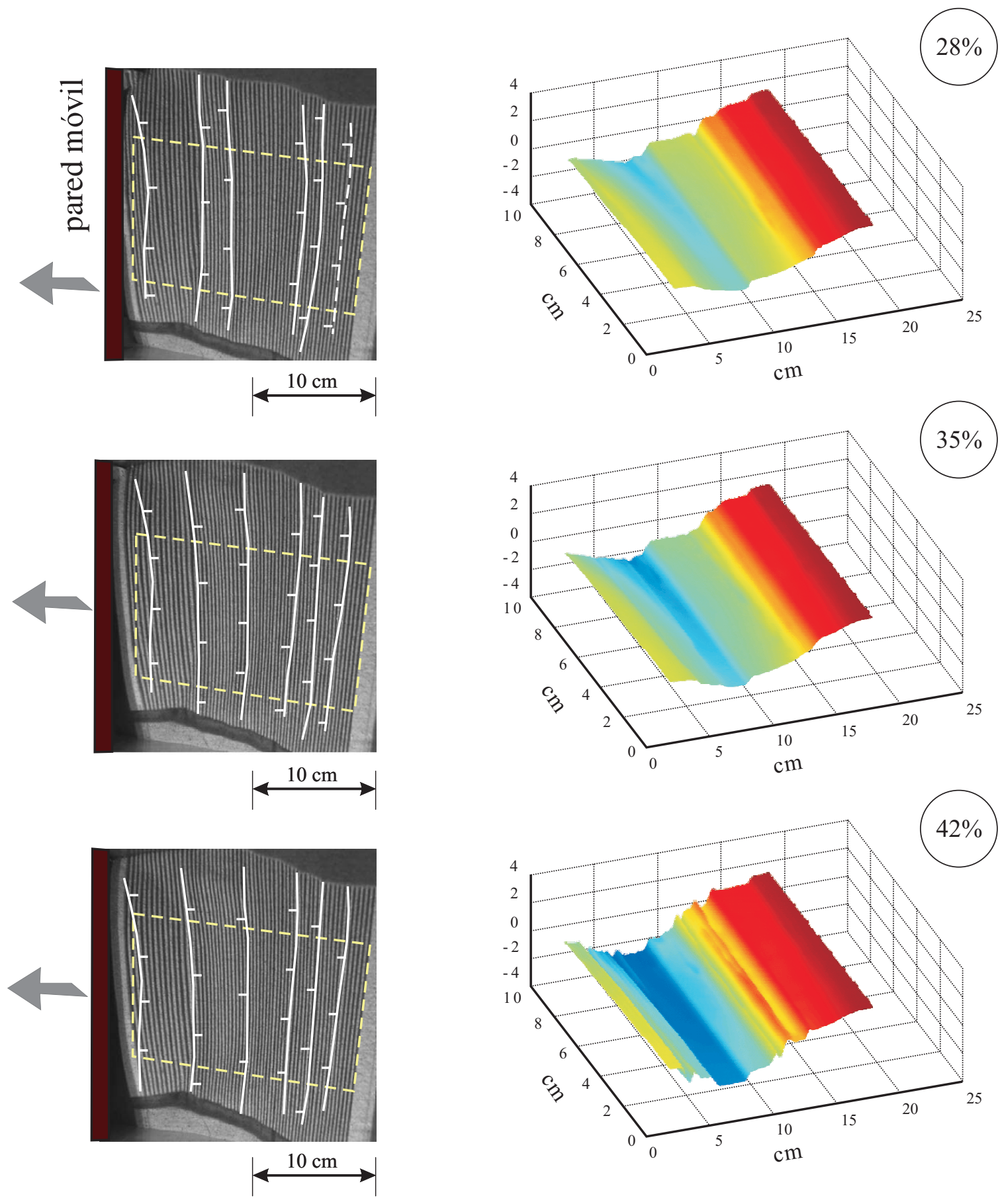

Simbolos

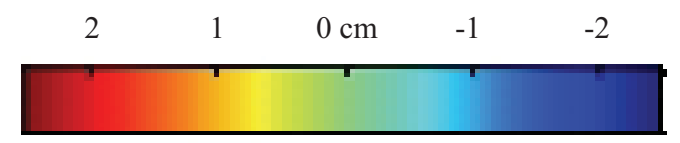

Figura 7b. Evolución de la deformación en el primer experimento, se muestra la interpretación tradicional en las fotografías obtenidas con un ángulo de $30^{\circ}$ y la vista del relieve obtenido en $3 \mathrm{D}$, entre 28 y $42 \%$ de extensión. 

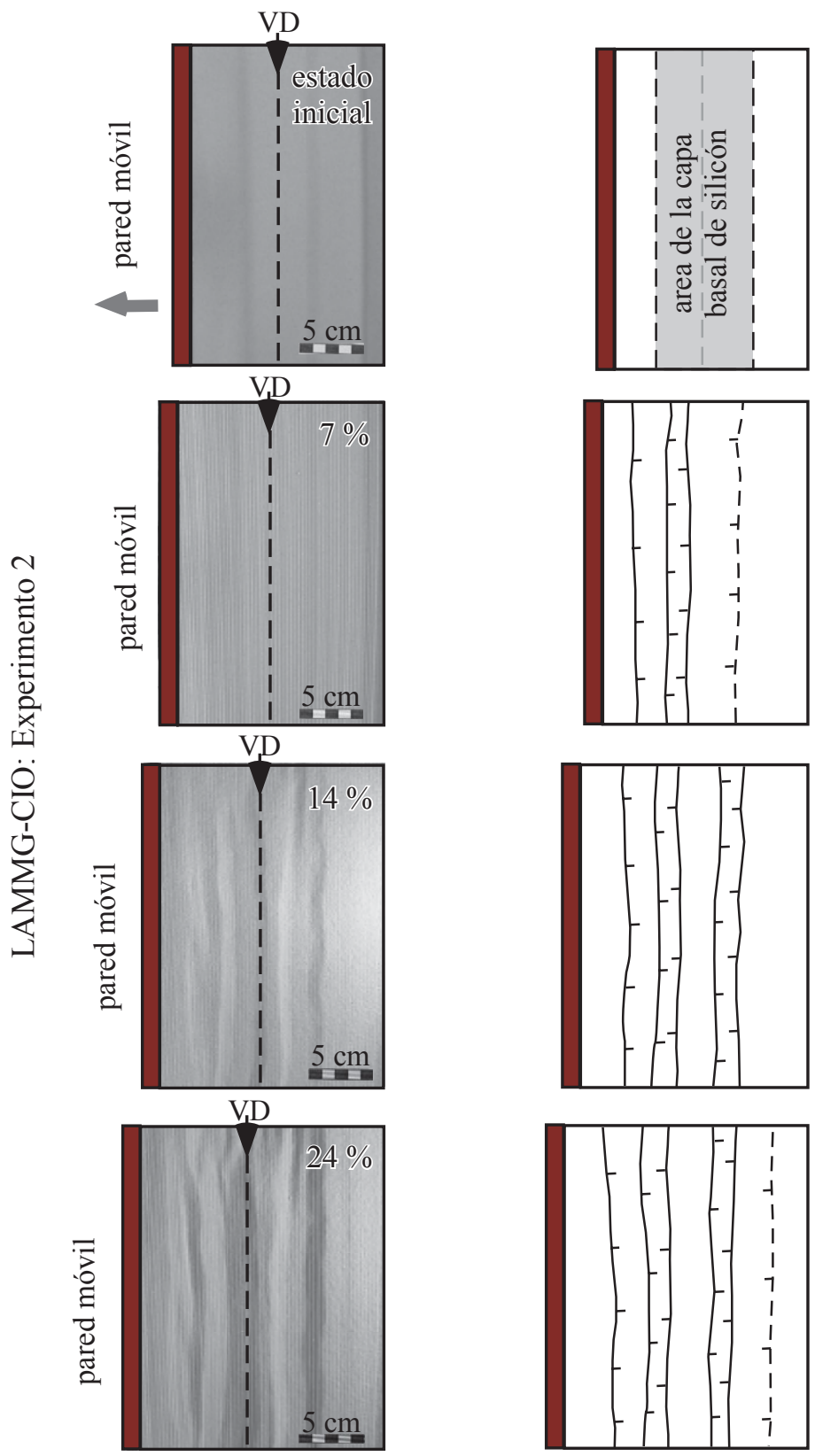

Figura 8. Evolución de la superficie en el segundo experimento, utilizando una capa débil y de baja viscosidad.

cm de profundidad y su zona de afectación alcanzó $6.5 \mathrm{~cm}$ de espesor, mientras que la fosa derecha alcanzó $1.3 \mathrm{~cm}$ de profundidad y $8.5 \mathrm{~cm}$ de espesor. En total el ancho de la zona extendida llegó a $16.5 \mathrm{~cm}$.

\subsection{Limitaciones de los modelos}

La evolución de la superficie durante el proceso de extensión en la naturaleza puede verse afectada por factores que no fueron considerados en los modelos; por ejemplo, el relieve original que existía antes de la deformación, la erosión y el depósito de sedimentos, la influencia de magmatismo, la evolución térmica de las cuencas, entre otros. En cuanto a sus condiciones de frontera, construcción y selección de materiales los modelos también presentan algunas limitaciones. Por ejemplo, los efectos de borde que genera la fricción en las paredes verticales en los modelos ocurren en la mayoría de los modelos. Para observar la sensibilidad de los modelos a los efectos de fricción en las fronteras laterales en el segundo experimento se examinaron dos condiciones diferentes: la pared izquierda de acrílico se cubrió con plástico transparente, mientras que la pared derecha fue confinada solo con arena. Como puede observarse en la Figura 9, la selección de las condiciones 


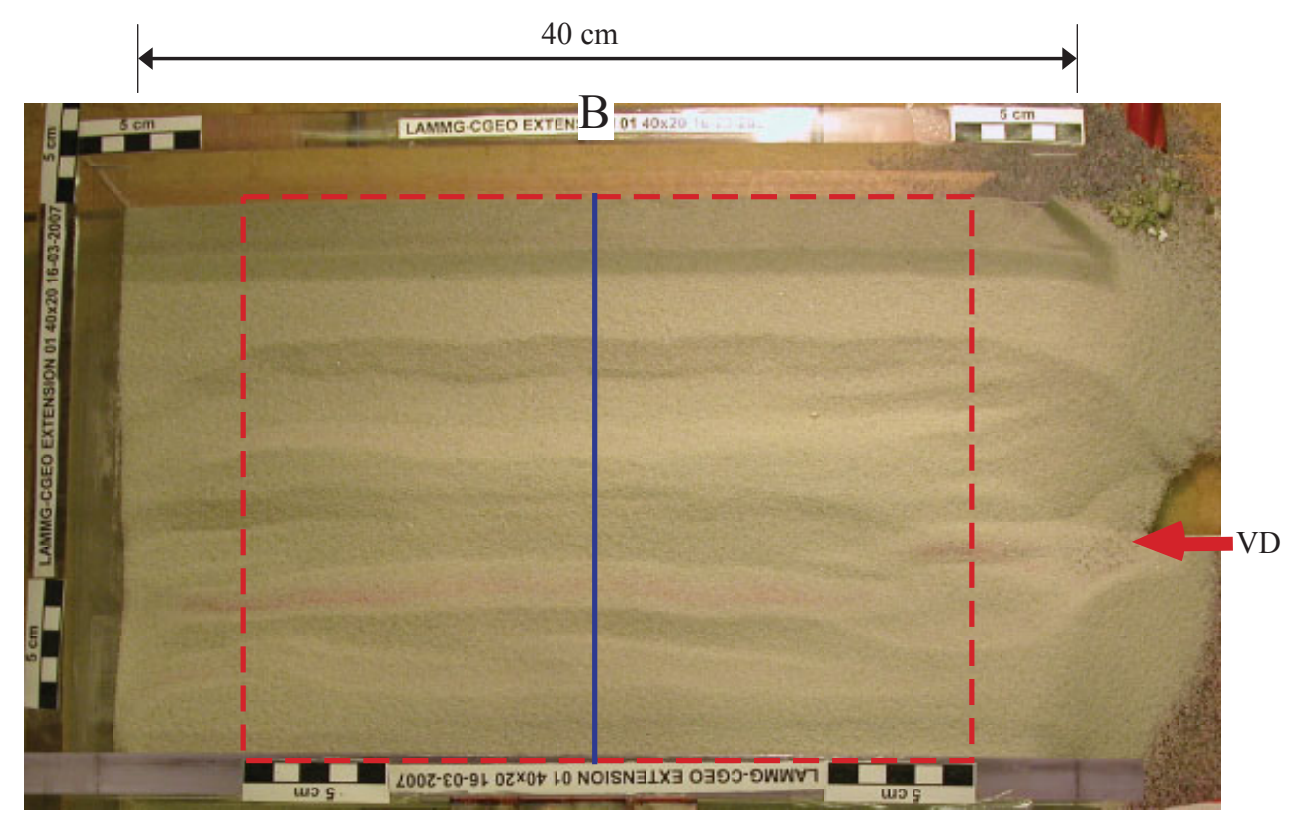

Figura 9. Fotografía del estado final de la deformación en el experimento de calibración, la selección de fronteras laterales diferentes cambian los efectos de frontera y su propagación hacia el interior del modelo. Del lado izquierdo, la frontera es una pared de acrílico mientras que del lado derecho solamente se añadió arena como confinante. El área señalada por la línea discontinua no presenta efectos de borde. La línea azul continua señala la posición de donde se realiza un corte para obtener una sección longitudinal B representativa, no presentada en este trabajo.

de frontera iniciales puede tener un efecto significativo en la forma y distribución de los efectos de borde. Para evitar estos efectos solo se analizó la deformación en el área cubierta por la línea roja punteada.

\section{Conclusiones}

La técnica de proyección de luz estructura ha arrojado resultados satisfactorios para reproducir de manera digital el relieve de la superficie durante la deformación de modelos analógicos. Sus características - buena resolución, bajo costo instrumental y no invasora - la hacen apropiada para este tipo de aplicaciones. El monitoreo y análisis de la superficie de los modelos para obtener un mapa de relieve digital de la superficie en modelos analógicos de deformación permite obtener información sobre la evolución de la deformación que no puede ser obtenida mediante el análisis tradicional de imágenes fotográficas, además de que permite trabajar con los resultados analógicos en un ambiente virtual. Se planea la realización de nuevos experimentos para explorar el uso conjunto de diferentes técnicas ópticas para trazar las trayectorias de partículas individuales durante la deformación en los modelos.

\section{Agradecimientos}

Este trabajo fue apoyado por los proyectos DGAPA PAPIIT (UNAM) IN120305 y CONACYT No. 46235 al
Laboratorio de Mecánica Multiescalar de Geosistemas (LAMMG), Centro de Geociencias. Agradecemos la ayuda de Dora Carreón-Freyre y Ricardo Carrizosa, quienes facilitaron equipo y colaboraron en la preparación de los materiales utilizados como análogos. Rodrigo Portillo participo en la preparación de un experimento. Teodoro HernándezTreviño del Instituto de Geofísica y Gustavo Tolson del Instituto de Geología, ambos de la UNAM participaron en la construcción del sistema de deformación. Daniel Malacara del CIO otorgo las facilidades para utilizar el laboratorio de Interferometría. Finalmente, agradecemos los comentarios y sugerencias de Peter Cobbold, Ana Crespo-Blanc y Jesús Blanco-García quienes revisaron el manuscrito.

\section{Referencias bibliográficas}

Barrientos, B., Cywiak, M., Lee, W. K., y Bryanston-Cross, P., 2004, Measurement of dynamic deformation using a superimposed grating: Revista Mexicana de Física, 50(1), 12-18.

Benes, V., y Davy, P., 1996, Modes of continental lithospheric extension: experimental verification of strain localization processes: Tectonophysics, 254, 69-87.

Bryanston-Cross, P. J., Quan, C., Judge, T. R., 1994, Application of the FFT method for the quantitative extraction of information from high resolution interferometric and photoelastic data: Optics and Laser Technology, 26(3), 147-155.

Byerlee, J. D., 1978, Friction of rocks: Pure and Applied Geophysics, 116, 615- 626 .

Corti, G., Bonini, M., Conticelli, S., Innocenti, F., Manetti, P., y Sokoutis, D., 2003, Analogue modelling of continental extension: a review focused on the relations between the patterns of deformation and the presence of magmas: Earth-Science Reviews, 1291, 1-79. 
Colletta, B., Letouzey, J., Pinedo, R., Ballard, J.F., y Balé, P., 1991, Computerized X-ray tomography analysis of sandbox models: Examples of thin-skinned thrust systems: Geology, 19, 1063-1067, doi: 10.1130/0091-7613(1991) 0192.3.CO;2.

Fischer, M. P., y Keating, D. P., 2005, Photogrammetric techniques for analyzing displacement, strain, and structural geometry in physical models: Application to the growth of monoclinal basement uplifts: GSA Bulletin, 117 (3-4), 369-382; doi: 10.1130/B25484.1.

Hubbert, M. K., 1937, Theory of scale models as applied to the study of geologic structures: Bulletin of the Geological Society of America, 48, 1459-1520.

Indebetouw, G., 1978, Profile measurement using projection of running fringes: Applied Optics, 17, 2930.

Muller, G., Mach, R., y Kaupper, K., 2001, Mapping of bridge pier scour with projection Moiré: Journal of Hydraulic Research, 39 (5), 1-7.

Persson, K., Garcia-Castellanos, D., y Sokoutis, D., 2004, River transport effects on compressional belts: First results from an integrated analogue-numerical model: Journal of Geophysical Research, 109, B01409, doi:10.1029/2002JB002274

Ranalli, G., 2001, Experimental tectonics: from Sir James Hall to the present: Journal of Geodynamics, 32 (1-2), $65-76$.

Ramberg, H., 1981., Gravity, Deformation and Earth's Crust: Academic, San Diego Calif., 452.

Schreurs, G., Hänni, R., y Vock, P., 2001, Four-dimensional analysis of analog models: Experiments on transfer zones in fold and thrust belts, in Koyi, H. A., and Mancktelow, N. S., eds., Tectonic modelling: A volume in Honor of Hans Ramberg: Boulder, Colorado: Geological Society of America Memoir, 193, 179-190.

Schreurs, G., Buiter, S. J. H., Boutelier, D., Corti, G., Costa, E., Cruden, A. R., Daniel, J-M., Hoth, S., Koyi, H., Kukowski, N., Lohrmann, J., Ravaglia, A., Schlische, R. W., Withjack, M. O., Yamada, Y., Cavozzi, C., Delventisette, C., Brady, J. A. E., Hoffmann-Rothe, A., Mengus, J-M., Montanari, D., y Nilforoushan, F., 2006, Analogue benchmarks of shortening and extension experiments., in Buiter,
S. J. H., and Scheurs, G. (eds). Analogue and numerical modeling of crustal-scale processes: Geological Society, London, Special Publications, 253, 1-27.

Srinivasan, E., Liu H. C., y Alioua, M., 1984, Automated phase-measuring profilometry of 3D diffuse objects: Applied Optics 23, 3105.

Takeda, M., y Mutoh, K., 1983, Fourier transform profilometry for the automatic measurement of 3-D object shapes: Applied Optics, 22, 3977.

Weijemars, R., y Schmeling, H., 1986, Scaling of Newtonian and nonNewtonian fluid dynamics without inertia for quantitative modeling of rock flow due to gravity (including the concept of rheological similarity): Physics of the Earth and Planetary Interiors, 43, 316-330.

Wilkerson, M.S., Marshak, S., y Bosworth, W., 1992, Computerized tomographic analysis of displacement trajectories and three-dimensional fold geometry above oblique thrust ramps: Geology, 20, 439-442.

Willingshofer, E., Sokoutis, D., y Burg, J-P., 2005, Lithospheric-scale analogue modeling of collision zones with a pre-existing weak zone. In: Gapais, D., Brun, J-P. y Cobbold, P. R. (eds). Deformation Mechanisms, Rheology and Tectonics: from Minerals to the Lithosphere: Geological Society, London, Special Publications, 243, 277-294.

Windecker, R., y Tiziani, H. J., 1995, Topometry of technical and biological objects by fringe projection: Applied Optics, 34, 3644.

Manuscrito recibido: Mayo 2, 2007

Manuscrito corregido recibido: Junio 21, 2007

Manuscrito aceptado: Agosto 6, 2007 